\title{
Nonlinear Control of an Active Magnetic Bearing with Output Constraint
}

\author{
Danh Huy Nguyen*, Tung Lam Nguyen*,**, Manh Linh Nguyen*, and Huy Phuong Nguyen* \\ ${ }^{*}$ School of Electrical Engineering, Hanoi University of Science and Technology \\ ** Institute for Control Engineering and Automation, Hanoi University of Science and Technology
}

\begin{tabular}{l}
\hline \hline Article Info \\
\hline Article history: \\
Received December 12, 2017 \\
Revised July 20, 2018 \\
Accepted Aug 9, 2018 \\
\hline
\end{tabular}

\section{Keyword:}

Active magnetic bearing

Backstepping control

Barrier Lyapunov function

Speed observer

\begin{abstract}
In this paper, an appropriate control strategy is proposed to handle the nonlinear dynamics of an active magnetic bearing (AMB). The goal of the control design is to drive the AMB rotor to the origin with improved transient response. In order to achieve this task, back stepping control technique with a barrier Lyapunov function are employed to keep the tracking error trajectory inside a predefined zone to avoid possible mechanical contact between rotor and stator. Besides, a speed observer is also used since information about rotor speed is not always available. The stability of the closed-loop system is proven. The effectiveness of the proposed control strategy is verified by numerical simulations.
\end{abstract}

Copyright (c) 2018 Institute of Advanced Engineering and Science. All rights reserved.

\section{Corresponding Author:}

Tung Lam Nguyen

School of Electrical Engineering, Hanoi University of Science and Technology

No 1, Dai Co Viet street, Hanoi, Vietnam

$+84989998384$

lam.nguyentung@hust.edu.vn

\section{INTRODUCTION}

Active magnetic bearing (AMB) has been developed in recent few decades to replace the conventional mechanical bearings. The main concept of an AMB is to use an electromagnetic force to support a body without any mechanical contact. In comparison with the traditional mechanical bearings with many drawbacks [1] and [2], AMB exhibits many advantages such as: frictionless, lubricant-free operation, active vibration control and unbalance compensation ability. Hence, AMB is a promising solution for high-speed applications such as high-speed motor [3], flywheel energy storage systems (FESS), .etc. For the proper operation of an AMB, rotor positioning control is a challenging task due to the inherent instability and nonlinearity of the system as mentioned in [4], [5], and [6]. In order to deal with system nonlinearity, authors of [7] propose a linearized AMB model and employed conventional PID control, similar approach can be found in [8]. In addition to linear control trend, an off-line tunning technique for centralized and decentralized linear AMB controllers are demonstrated in [9]. When the variation of the rotor position is small, linearizing the model in a small region around an equilibrium point in order to use the linear control technique is an appropriate approach to stabilize the AMB system. However, the performance of the positioning system may degrade significantly when the operating point is far from the desired equilibrium point. Hence, nonlinear control technique has been studied to further improve the systems performance.

In order to stabilize rotor when facing with a vibrating base, [10] design a sliding mode scheme for an AMB system. The closed-loop system exhibits robustness to uncertainties and external disturbances. Based on Lyapunov's direct method and the singular perturbation order-reduction technique, the state feedback control is derived in [11]. Through a set of numerical simulations, the state feedback control shows its strength in comparison with output feedback and deadbeat controls. Inspired by loop shaping properties of $H_{\infty}$ optimization and disturbances rejection ability of the disturbance observer-based controller, [12] successfully develop a hybrid controller whose effectiveness are verified via simulations and real-time experiments. By investigating strong nonlinearity of 3-poles AMB, a feedback linearization law is composed in [13]. Rotor responses also are restricted to a safe distance from stator boundary, however, the requirement of initial conditions might be difficult 
to archive in practice. In a circumstance of the AMB driving rotor with unknown mass imbalance, [14] proposes an adaptive mechanism to tackle this situation. Taking voltage input saturation into account, a robust fuzzy control that is able to stabilize the AMB rotor is developed in [15]. Apart from conventional AMB, [16] suggests a design and decoupling control for axial AMB by combining optimal algorithm and feed-forward control.

The paper proposes an nonlinear control approach to stabilizing AMB rotor problem based on backstepping control. A speed observer is utilized in this research to acquire rotor speed information. Barrier Lyapunov candidate function is embedded into design process to drive the AMB rotor trajectory inside a defined range. The stability of the closed-loop system is proven. A set of numerical simulations are given to verify the control effectiveness.

\section{MATHEMATICAL MODEL OF AMB}

Figure 1 shows the one degree of freedom (1DOF) AMB system considered in this paper. In this figure,

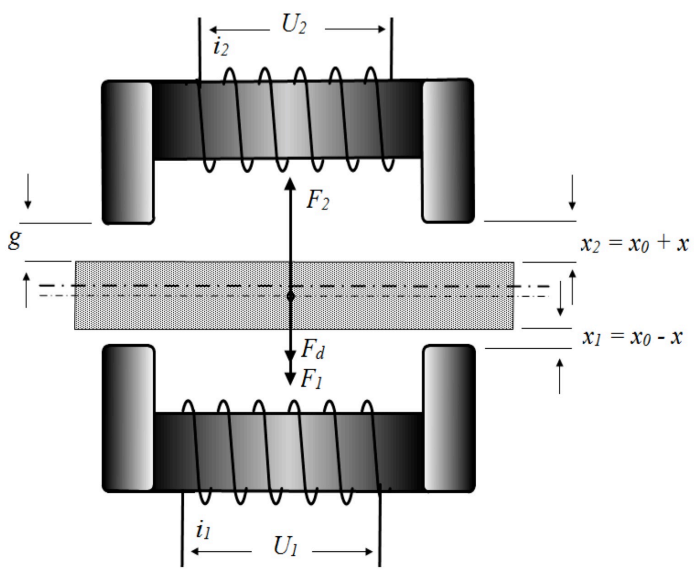

Figure 1. An AMB system.

$F_{k}, u_{k}$ and $i_{k}$ are the electromagnetic force, applied input voltage and current of the corresponding coil $k$, respectively, with $k=1,2 ; F_{d}$ is the disturbance force caused by rotor mass and other uncertainties. Suppose that the displacement $x$ of the rotor from the nominal position $x_{0}$ caused by the initial voltage and current $\left(u_{0}, i_{0}\right)$. Denote $x_{1}$ and $x_{2}$ are the air gaps between the rotor and the left and right side stators, it yields

$$
\begin{aligned}
x_{1} & =x_{0}-x, \quad x_{2}=x_{0}+x \\
i_{1} & =i_{0}-i, \quad i_{2}=i_{0}+i \\
u_{1} & =u_{0}-u, \quad u_{2}=u_{0}+u
\end{aligned}
$$

By using (1)-(3), a fundamental operation gives

$$
\left\{\begin{array}{l}
\frac{d x}{d t}=v \\
\frac{d v}{d t}=\frac{K}{4 m}\left(\frac{i_{1}}{x_{0}-x}\right)^{2}-\frac{K}{4 m}\left(\frac{i_{2}}{x_{0}+x}\right)^{2}+\frac{F_{d}}{m} \\
\frac{d i_{1}}{d t}=A\left[-R i_{1}-\frac{K}{2\left(x_{0}-x\right)} v i_{1}+u_{1}\right] \\
\frac{d i_{2}}{d t}=B\left[-R i_{2}+\frac{K}{2\left(x_{0}+x\right)} v i_{2}+u_{2}\right]
\end{array}\right.
$$

where,

$$
A=\frac{2\left(x_{0}-x\right)}{2 L_{s}\left(x_{0}-x\right)+K}, \quad B=\frac{2\left(x_{0}+x\right)}{2 L_{s}\left(x_{0}+x\right)+K}, \quad K=\mu_{g} N^{2} A_{g}
$$

In (5), $\mu_{g}$ is the permeability of air, $N$ is the number of turns in each coil and $A_{g}$ is the cross-section area of the electromagnet. Due to the fact that only the position of the rotor and the two current $i_{1}$ and $i_{2}$ of the corresponding coils are available for measurement, a speed observer is needed to estimate the rotor speed. In this research, the nonlinear observer proposed in [17] is utilized for speed estimation as follows

$$
\hat{v}=\eta+\theta \lambda+k x+\frac{F_{d}}{m} \frac{1}{k}
$$


where,

$$
\begin{aligned}
& \dot{\eta}=-k \eta-k^{2} x \\
& \dot{\lambda}=-k \lambda+\frac{i_{1}^{2}}{\left(x_{0}-x\right)^{2}}-\frac{i_{2}^{2}}{\left(x_{0}+x\right)^{2}} \\
& \theta=\frac{K}{4 m}
\end{aligned}
$$

where $k$ is a positive constant. Suppose that the initial conditions of the system is

$$
\eta(0)=0, \quad \lambda(0)=0
$$

Denote the speed estimation error $\epsilon$ as

$$
\epsilon=v-\hat{v}
$$

Then, by differentiating both side of (11) and based on (4) and (6)-(9), it gives

$$
\begin{aligned}
\dot{\epsilon} & =\dot{v}-\dot{\hat{v}} \\
& =\theta\left(\frac{i_{1}}{x_{0}-x}\right)^{2}-\theta\left(\frac{i_{2}}{x_{0}+x}\right)^{2}+\frac{F_{d}}{m}-\dot{\eta}-\theta \dot{\lambda}-k \dot{x} \\
& =k\left(\eta+\theta \lambda+k x+\frac{1}{k} \frac{F_{d}}{m}-v\right) \\
& =-k \epsilon
\end{aligned}
$$

Relation (12) means that the speed estimation error $\epsilon$ exponentially converges to zero and the convergence speed depends on $k$. By using the speed observer (6), the state space model of the AMB system can be rewritten as

$$
\left\{\begin{array}{l}
\hat{\dot{x}}=\hat{v}+\epsilon \\
\hat{v}=\eta+\theta \lambda+k x+\frac{1}{k} \frac{F_{d}}{m} \\
\dot{\lambda}=-k \lambda+\theta\left(\frac{i_{1}}{x_{0}-x}\right)^{2}-\theta\left(\frac{i_{2}}{x_{0}+x}\right)^{2} \\
\dot{\eta}=-k \eta-k x^{2} \\
\dot{i_{1}}=A\left[-R i_{1}-\frac{K}{2\left(x_{0}-x\right)}(\hat{v}+\epsilon) i_{1}+u_{1}\right] \\
\dot{i}_{2}=B\left[-R i_{2}+\frac{K}{2\left(x_{0}+x\right)}(\hat{v}+\epsilon) i_{2}+u_{2}\right]
\end{array}\right.
$$

\section{CONTROL DESIGN}

It can be observed from (13) that the model of the AMB has the form of a strict-feedback system. Hence, backstepping control technique is chosen to stabilize the system. The control design is implemented step-by-step as follows.

Step 1: The goal of the control design in this step is to drive the rotor to a desired position $y_{r}$ with minimized tracking error. Denote $z_{1}$ as the position error

$$
z_{1}=x-y_{r}
$$

Differentiating both side of (14) gives

$$
\begin{aligned}
\dot{z}_{1} & =\dot{x}-\dot{y}_{r} \\
& =\eta+\theta \lambda+k\left(z_{1}+y_{r}\right)+\frac{g}{k}+\epsilon-\dot{y}_{r}
\end{aligned}
$$

Consider the following barrier Lyapunov candidate function

$$
V_{1}=\frac{1}{2} \log \left(\frac{k_{b}^{2}}{k_{b}^{2}-z_{1}^{2}}\right)+\frac{1}{2 k d_{1}} \epsilon
$$

with $d_{1}>0$. By using the proposed barrier Lyapunov function, the position error $z_{1}$ is kept in a region restricted by $\left(-k_{b}, k_{b}\right)$ where $k_{b}$ is a positive number. This means the overshoot of the AMB in transient state can be 
handle by $k_{b}$. This property is meaningful in practice since the mechanical contact between the rotor and stator can be avoided. Differentiating both sides of (16), it gives

$$
\begin{aligned}
\dot{V}_{1} & =\frac{z_{1} \dot{z}_{1}}{k_{b}^{2}-z_{1}^{2}}-\frac{\epsilon^{2}}{d_{1}} \\
& =\frac{z_{1}\left(\eta+\theta \lambda+k z_{1}+\epsilon-\dot{Y}_{r}+k Y_{r}\right)}{k_{b}^{2}-z_{1}^{2}}-\frac{\epsilon^{2}}{d_{1}}
\end{aligned}
$$

in order to render $\dot{V}_{1} \leq 0$, the virtual control action $\lambda_{v}$ is selected as

$$
\lambda_{v}=\frac{1}{\theta}\left[-c_{1} z_{1}\left(k_{b}^{2}-z_{1}^{2}\right)-\frac{d_{1} z_{1}}{k_{b}^{2}-z_{1}^{2}}-k\left(z_{1}+Y_{r}\right)+\dot{Y}_{r}\right]
$$

with $c_{1}>0$.

Step 2: As seen in the previous step, if $\lambda=\lambda_{v}$, then $\dot{V}_{1} \leq 0$ which results in $\epsilon=0$ and $z_{1}=0$. Hence, the goal of this step is to guarantee that $\lambda$ approaches to $\lambda_{v}$. Denote $z_{2}$ as

$$
z_{2}=\lambda-\lambda_{v}
$$

The candidate Lyapunov function is chosen as

$$
V_{2}=\frac{1}{2} \log \left(\frac{k_{b}^{2}}{k_{b}^{2}-z_{1}^{2}}\right)+\frac{1}{2} z_{2}^{2}+\frac{\epsilon^{2}}{2 k}\left(\frac{1}{d_{1}}+\frac{1}{d_{2}}\right)
$$

with $d_{2}>0$. Differentiating both sides of (20) gives

$$
\dot{V}_{2}=\frac{z_{1} \dot{z}_{1}}{k_{b}^{2}-z_{1}^{2}}+z_{2} \dot{z}_{2}-\epsilon^{2}\left(\frac{1}{d_{1}}+\frac{1}{d_{2}}\right)
$$

Taking $\lambda$ from (17) and substitute it into (15) give

$$
\dot{z}_{1}=\theta z_{2}-c_{1} z_{1}\left(k_{b}^{2}-z_{1}^{2}\right)-\frac{d_{1} z_{1}}{k_{b}^{2}-z_{1}^{2}}+\epsilon
$$

Differentiating both sides of (19) gives

$$
\dot{z}_{2}=\dot{\lambda}-\frac{\partial \lambda_{v}}{\partial z_{1}} \hat{v}+\epsilon-\dot{Y}_{r}-\frac{\partial \lambda_{v}}{\partial \eta}-\frac{\ddot{Y}_{r}}{\theta}+\frac{k \dot{Y}_{r}}{\theta}
$$

in which,

$$
\begin{aligned}
& \frac{\partial \lambda_{v}}{\partial z_{1}}=\frac{1}{\theta}\left[-k-c_{1} k_{b}^{2}+3 c_{1} z_{2}^{2}-\frac{d_{1}\left(k_{b}^{2}+z_{1}^{2}\right)}{\left(k_{b}^{2}-z_{1}^{2}\right)^{2}}\right] \\
& \frac{\partial \lambda_{v}}{\eta}=-\frac{1}{\theta}
\end{aligned}
$$

Then, by substituting (22) and (23) into (21), it results in

$$
\begin{aligned}
\dot{V}_{2}= & -c_{1} z_{1}-d_{1}\left(\frac{z_{1}}{k_{b}^{2}-z_{1}^{2}}-\frac{\epsilon}{2 d_{1}}\right)^{2}-\epsilon^{2}\left(\frac{1}{d_{2}}+\frac{3}{4 d_{1}}\right) \\
& +z_{2}\left(\frac{\theta z_{1}}{k_{b}^{2}-z_{1}^{2}}+\dot{\lambda}-\frac{\partial \lambda_{v}}{\partial \eta} \dot{\eta}-\frac{\partial \lambda_{v}}{\partial z_{1}} \dot{z}_{1}-\frac{\ddot{Y}_{r}}{\theta}+\frac{k \dot{Y}_{r}}{\theta}\right)
\end{aligned}
$$

From (26), to guarantee that $\dot{V}_{2}<0$, the virtual control signal $\dot{\lambda}_{v}$ is chosen as

$$
\dot{\lambda}_{v}=-c_{2} z_{2}+\frac{\partial \lambda_{v}}{\partial \eta} \dot{\eta}+\frac{\partial \lambda_{v}}{\partial z_{1}}\left(\hat{v}-\dot{Y}_{r}\right)-d_{2} z_{2} \frac{\partial \lambda_{v}{ }^{2}}{\partial z_{1}}-\frac{\theta z_{1}}{k_{b}^{2}-z_{1}^{2}}+\frac{\ddot{Y}_{r}}{\theta}-\frac{k \dot{Y}_{r}}{\theta}
$$

with $c_{2}>0$. If

$$
\dot{\lambda}=\dot{\lambda}_{v}
$$


then $\dot{V}_{2}<0$. However, the solution for (27) can not be obtained since it depends on two variables $i_{1}$ and $i_{2}$. In oder to solve the abovementioned problem and to save the energy, a switching control strategy is employed in which either $i_{1}$ or $i_{2}$ is used. If $x<0$, coil 2 is turned off which means $i_{2}=0$, then

$$
\dot{\lambda}=-k \lambda+\frac{i_{1}^{2}}{\left(x_{0}-x\right)^{2}}
$$

Substitute (28) into (27), the desired current of coil 1 is

$$
i_{1}=i_{1, d}=x_{0}-x \sqrt{\dot{\lambda}_{d}+k \lambda}
$$

Denote $\alpha=\dot{\lambda}_{d}+k \lambda$, it gives

$$
i_{1}=i_{1, d}=x_{0}-x \sqrt{\alpha}
$$

Similarly, if $x \geq 0$, then $i_{1}=0$ and hence

$$
\dot{\lambda}=-k \lambda-\frac{i_{2}^{2}}{\left(x_{0}+x\right)^{2}}
$$

The reference current for coil 2 is

$$
i_{2}=i_{2, d}=x_{0}+x \sqrt{-\alpha}
$$

Remark: Equations (28), (31), and the definition of $\alpha$ indicate that square root operations in (29) and (32) always hold.

Step 3: The goal of this final step is to obtain $u_{1}$ and $u_{2}$ which drives $i_{1}$ and $i_{2}$ to their desired values $i_{1, d}$ and $i_{2, d}$, respectively. Case 1: $x<0$. Denote $z_{3}$ as

$$
z_{3}=i_{1}-i_{1, d}
$$

Differentiate (33), it gives

$$
\begin{aligned}
\dot{z}_{3}= & \dot{i}_{1}-\dot{i}_{1, d} \\
= & -A R i_{1}+\left[-\frac{A K i_{1}}{2\left(x_{0}-x\right)^{2}}-\frac{\partial i_{1, d}}{\partial z_{1}}(\hat{v}+\epsilon)-\dot{Y}_{r}\right] \\
& +A u_{1}-\frac{\partial i_{1, d}}{\partial \lambda} \dot{\lambda}-\frac{\partial i_{1, d}}{\partial \dot{Y}_{r}} \ddot{Y}_{r}-\frac{\partial i_{1, d}}{\partial \ddot{Y}_{r}} \dddot{Y}_{r}-\left[\frac{A K i_{1}}{2\left(x_{0}-x\right)^{2}}+\frac{\partial i_{1, d}}{\partial Y_{r}}\right] \dot{Y}_{r}
\end{aligned}
$$

in which

$$
\begin{aligned}
& \frac{\partial i_{1, d}}{\partial z_{1}}=-\sqrt{\alpha}+\left(x_{0}-x\right) \frac{1}{2 \sqrt{\alpha}} \frac{\partial \alpha}{\partial z_{1}} \\
& \frac{\partial i_{1, d}}{\partial \lambda}=\left(x_{0}-x\right) \frac{1}{2 \sqrt{\alpha}} \frac{\partial \alpha}{\partial \lambda} \\
& \frac{\partial i_{1, d}}{\partial \eta}=\left(x_{0}-x\right) \frac{1}{2 \sqrt{\alpha}} \frac{\partial \alpha}{\partial \eta} \\
& \frac{\partial i_{1, d}}{\partial Y_{r}}=-\sqrt{\alpha}+\left(x_{0}-x\right) \frac{1}{2 \sqrt{\alpha}} \frac{\partial \alpha}{\partial Y_{r}} \\
& \frac{\partial i_{1, d}}{\partial \dot{Y}_{r}}=\left(x_{0}-x\right) \frac{1}{2 \sqrt{\alpha}} \frac{\partial \alpha}{\partial \dot{Y}_{r}} \\
& \frac{\partial i_{1, d}}{\partial \ddot{Y}_{r}}=\left(x_{0}-x\right) \frac{1}{2 \sqrt{\alpha}} \frac{\partial \alpha}{\partial \ddot{Y}_{r}}
\end{aligned}
$$


with,

$$
\begin{aligned}
\frac{\partial \alpha}{\partial \eta}= & -\left(\frac{\partial \lambda_{v}}{\partial z_{1}}\right)^{2} \frac{d_{2}}{\theta}-\frac{c_{2}}{\theta}+\frac{\partial \lambda_{v}}{\partial z_{1}}+\frac{k}{\theta} \\
\frac{\partial \alpha}{\partial \lambda}= & -\left(\frac{\partial \lambda_{v}}{\partial z_{1}}\right)^{2} d_{2}-c_{2}+k+\theta \frac{\partial \lambda_{v}}{\partial z_{1}} \\
\frac{\partial \lambda}{\partial Y_{r}}= & c_{2}\left(\frac{-k}{\theta}\right)+d_{2}\left(\frac{\partial \lambda_{v}}{\partial z_{1}}\right)^{2}\left(\frac{-k}{\theta}\right)+\frac{\partial \lambda_{v}}{\partial z_{1}} k \\
\frac{\partial \alpha}{\partial \dot{Y}_{r}}= & c_{2} \frac{1}{\theta}+d_{2}\left(\frac{\partial \lambda_{v}}{\partial z_{1}}\right)^{2} \frac{1}{\theta}-\frac{\partial \lambda_{v}}{\partial z_{1}}-\frac{k}{\theta} \\
\frac{\partial \alpha}{\partial \ddot{Y}_{r}}= & \frac{1}{\theta} \\
\frac{\partial \alpha}{\partial z_{1}}= & \frac{\partial^{2} \lambda_{v}}{\partial z_{1}^{2}} \hat{v}+c_{2} \frac{\partial \lambda_{v}}{\partial z_{1}}+d_{2}\left(\frac{\partial \lambda_{v}}{\partial z_{3}}\right)^{3}+k \frac{\partial \lambda_{v}}{\partial z_{1}} \\
& +\frac{k^{2}}{\theta}+2 d_{2}\left(\lambda_{v}-\lambda\right) \frac{\partial \lambda_{v}}{\partial z_{1}} \frac{\partial^{2} \lambda_{v}}{\partial z^{2}}-\frac{\theta\left(k_{b}^{2}+z_{1}^{2}\right)}{\left(k_{b}^{2}-z_{1}^{2}\right)^{2}} \\
\frac{\partial^{2} \lambda_{v}}{\partial z_{1}^{2}}= & \frac{1}{\theta}\left[6 c_{1} z_{1}-\frac{2 z_{1} d_{1}\left(3 k_{b}^{2}+z_{1}^{2}\right)}{\left(k_{b}^{2}-z_{1}^{2}\right)^{3}}\right]
\end{aligned}
$$

The third Lyapunov candidate function is chosen as

$$
V_{3}=V_{2}+\frac{1}{2} z_{3}^{2}+\frac{\epsilon^{2}}{2 k d_{3}}
$$

with $d_{3}>0$. The differentiation of (48) is

$$
\dot{V}_{3}=-c_{1} z_{1}^{2}-c_{2} z_{2}^{2}+z_{3} \dot{z}_{3}-\epsilon^{2}\left(\frac{3}{4 d_{1}}+\frac{3}{4 d_{2}}+\frac{1}{d_{3}}\right)-d_{1}\left(\frac{z_{1}}{k_{b}^{2}-z_{1}^{2}}-\frac{\epsilon}{2 d_{1}}\right)^{2}-d_{2}\left(z_{2} \frac{\partial \lambda_{v}}{\partial z_{1}}-\frac{\epsilon}{2 d_{2}}\right)^{2}
$$

To make $\dot{V}_{3} \leq 0, \dot{z}_{3}$ is chose as

$$
\dot{z}_{3}=-c_{3} z_{3}-d_{3} z_{3} F^{2}+\epsilon F
$$

where

$$
F=-\frac{A K i_{1}}{2\left(x_{0}-x\right)^{2}}-\frac{\partial i_{1, d}}{\partial z_{1}}
$$

and $c_{3}>0$. The control signal $u_{1}$ which stabilizes the AMB system around the equilibrium point $x_{0}$ in Case 1 can be obtained from (34) and (50) as follows

$$
\begin{aligned}
u_{1}= & \frac{1}{A}\left[A R i_{1}-F \hat{v}-\dot{Y}_{r}-c_{3} z_{3}-d_{3} z_{3} F^{2}+\frac{\partial i_{1, d}}{\partial \lambda} \dot{\lambda}+\frac{\partial i_{1, d}}{\partial \eta} \dot{\eta}\right] \\
& +\frac{1}{A}\left[\left(\frac{A K i_{1}}{2\left(x_{0}-x\right)^{2}}+\frac{\partial i_{1, d}}{\partial Y_{r}}\right) \dot{Y}_{r}+\frac{\partial i_{1, d}}{\dot{Y}_{r}} \ddot{Y}_{r}+\frac{\partial i_{1, d}}{\partial \ddot{Y}_{r}} \dddot{Y}_{r}\right]
\end{aligned}
$$

Case 2: In this case,

$$
x>0, \quad i_{1}^{2}=0, u_{1}=0, \quad 2, d=x_{0}+x \sqrt{-\alpha}
$$

Similar to Case 1 , the control action $u_{2}$ in this case is

$$
\begin{aligned}
u_{2}= & \frac{1}{B}\left[B R i_{2}-G \hat{v}-\dot{Y}_{r}-c_{4} z_{4}-d_{4} z_{4} F^{2}+\frac{\partial i_{2, d}}{\partial \lambda} \dot{\lambda}\right] \\
& +\frac{1}{B}\left[\frac{\partial i_{2, d}}{\partial \eta} \dot{\eta}-\left(\frac{B K i_{2}}{2\left(x_{0}+x\right)^{2}}-\frac{\partial i_{2, d}}{\partial Y_{r}}\right) \dot{Y}_{r}+\frac{\partial i_{2, d}}{\partial \dot{Y}_{r}} \ddot{Y}_{r}\right]+\frac{1}{B}\left[\frac{\partial i_{2, d}}{\partial \ddot{Y}_{r}} \dddot{Y}_{r}\right]
\end{aligned}
$$

with $c_{4}>0$ and

$$
G=\frac{B K i_{2}}{2\left(x_{0}+x\right)^{2}}-\frac{\partial i_{2, d}}{\partial z_{1}}
$$




\section{STABILITY ANALYSIS}

Similar to the control design section, the stability analysis of the AMB control system is also divided into two cases.

Case 1: $x<0$ and $i_{2}=0$. The closed-loop system can be described by

$$
\left\{\begin{array}{l}
\dot{z}_{1}=\theta z_{2}-c_{1} z_{1}\left(k_{b}^{2}-z_{1}^{2}\right)-\frac{d_{1} z_{1}}{k_{b}^{2}-z_{1}^{2}}+\epsilon \\
\dot{z}_{2}=-c_{2} z_{2}-d_{2} z_{2}\left(\frac{\partial \lambda_{v}}{\partial z_{1}}\right)-\frac{\theta z_{1}}{k_{b}^{2}-z_{1}^{2}}-\frac{\partial \lambda_{v}}{\partial z_{1}} \epsilon \\
\dot{z}_{3}=\dot{i}_{1}-\dot{i}_{1, d}
\end{array}\right.
$$

where,

$$
\begin{aligned}
\dot{i}_{1} & =A\left[-R i_{1}-\frac{K(\hat{v}+\epsilon) i_{1}}{2\left(x_{0}-x\right)^{2}}+u_{1}\right] \\
\dot{i}_{1, d} & =\frac{\partial i_{1, d}}{\partial z_{1}} \dot{z}_{1}+\frac{\partial i_{1, d}}{\partial \lambda} \dot{\lambda}+\frac{\partial i_{1, d}}{\partial \eta} \dot{\eta}+\frac{\partial i_{1, d}}{\partial Y_{r}} \dot{Y}_{r}+\frac{\partial i_{1, d}}{\partial \dot{Y}_{r}} \ddot{Y}_{r}+\frac{\partial i_{1, d}}{\partial \ddot{Y}_{r}} \dddot{Y}_{r} \\
x & =z_{1}+Y_{r}
\end{aligned}
$$

The velocity observer in this case is

$$
\hat{v}=\eta+\theta \lambda+k x+\frac{g}{k}
$$

with,

$$
\left\{\begin{array}{l}
\dot{\eta}=-k \eta-k^{2} x \\
\dot{\lambda}=-k \lambda-\frac{i_{2}^{2}}{\left(x_{0}+x\right)^{2}}
\end{array}\right.
$$

and

$$
\begin{aligned}
& \frac{\partial \alpha}{\partial \eta}=-\left(\frac{\partial \lambda_{v}}{\partial z_{1}}\right)^{2} \frac{d_{2}}{\theta}-\frac{c_{2}}{\theta}+\frac{\partial \lambda_{v}}{\partial z_{1}}+\frac{k}{\theta} \\
& \frac{\partial \alpha}{\partial \lambda}=-\left(\frac{\partial \lambda_{v}}{\partial z_{1}}\right)^{2} d_{2}-c_{2}+k+\theta \frac{\partial \lambda_{v}}{\partial z_{1}} \\
& \frac{\partial \alpha}{\partial Y_{r}}=-c_{2} \frac{k}{\theta}-d_{2}\left(\frac{\partial \lambda_{v}^{2}}{\partial z_{1}}\right) \frac{k}{\theta}+\frac{\partial \lambda_{v}}{\partial z_{1}} k \\
& \frac{\partial \alpha}{\partial \dot{Y}_{r}}=\frac{c_{2}}{\theta}+d_{2}\left(\frac{\partial \lambda_{v}}{\partial z_{1}}\right)^{2} \frac{1}{\theta}-\frac{\partial \lambda_{v}}{\partial z_{1}}-\frac{k}{\theta} \\
& \frac{\partial \alpha}{\ddot{Y}_{r}}=\frac{1}{\theta} \\
& \frac{\partial \alpha}{\partial z_{1}}=\frac{\partial^{2} \lambda_{v}}{\partial z_{1}^{2}} \hat{v}+c_{2} \frac{\partial \lambda_{v}}{\partial z_{1}}+d_{2}\left(\frac{\partial \lambda_{v}}{\partial z_{1}}\right)^{3}+k \frac{\partial \lambda_{v}}{\partial z_{1}} \\
& +\frac{k^{2}}{\theta}+2 d_{2}\left(\lambda_{v}-\lambda\right) \frac{\partial \lambda_{v}}{\partial z_{1}} \frac{\partial^{2} \lambda_{v}}{\partial z^{2}}-\frac{\theta\left(k_{b}^{2}+z_{1}^{2}\right)}{\left(k_{b}^{2}-z_{1}^{2}\right)^{2}} \\
& \frac{\partial^{2} \lambda_{v}}{\partial z_{1}^{2}}=\frac{1}{\theta}\left[6 c_{1} z_{1}-\frac{2 z_{1} d_{1} 3 k_{b}^{2}+z_{1}^{2}}{\left(k_{b}^{2}-z_{1}^{2}\right)^{3}}\right] \\
& \frac{\partial i_{1, d}}{\partial z_{1}}=-\sqrt{\alpha}+\left(x_{0}-x\right) \frac{1}{2 \sqrt{\alpha}} \frac{\partial \alpha}{\partial z_{1}} \\
& \frac{\partial i_{1, d}}{\partial \lambda}=\left(x_{0}-x\right) \frac{1}{2 \sqrt{\alpha}} \frac{\partial \alpha}{\partial \lambda} \\
& \frac{\partial i_{1, d}}{\partial \eta}=\left(x_{0}-x\right) \frac{1}{2 \sqrt{\alpha}} \frac{\partial \alpha}{\partial \eta} \\
& \frac{\partial i_{1, d}}{\partial Y_{r}}=-\sqrt{\alpha}+\left(x_{0}-x\right) \frac{1}{2 \sqrt{\alpha}} \frac{\partial \alpha}{\partial Y_{r}}
\end{aligned}
$$




$$
\begin{aligned}
& \frac{\partial i_{1, d}}{\partial \dot{Y}_{r}}=\left(x_{0}-x\right) \frac{1}{2 \sqrt{\alpha}} \frac{\partial \alpha}{\partial \dot{Y}_{r}} \\
& \frac{\partial i_{1, d}}{\partial \ddot{Y}_{r}}=\left(x_{0}-x\right) \frac{1}{2 \sqrt{\alpha}} \frac{\partial \alpha}{\partial \ddot{Y}_{r}}
\end{aligned}
$$

To show the stability of the AMB system in this case, consider the following candidate Lyapunov function

$$
V_{C 1}=\frac{1}{2} \log \left(\frac{k_{b}^{2}}{k_{b}^{2}-z_{1}^{2}}\right)+\frac{1}{2} z_{2}^{2}+\frac{1}{2} z_{3}^{2}+\frac{\epsilon^{2}}{2 k}\left(\frac{1}{d_{1}}+\frac{1}{d_{2}}+\frac{1}{d_{3}}\right)
$$

Differentiate both sides of (75) yields

$$
\begin{aligned}
\dot{V}_{C 1}= & -c_{1} z_{1}^{2}-c_{2} z_{2}^{2}-c_{3} z_{3}^{2}-d_{1}\left(\frac{z_{1}}{k_{b}^{2}-z_{1}^{2}}-\frac{\epsilon}{2 d_{1}}\right)^{2} \\
& -d_{2}\left(\frac{\partial \lambda_{v}}{\partial z_{1}}-\frac{\epsilon}{2 d_{2}}\right)^{2}-d_{3}\left(z_{3} F-\frac{\epsilon}{2 d_{3}}\right)^{2}-\left(\frac{3}{4 d_{1}}+\frac{3}{4 d_{2}} \frac{3}{4 d_{3}}\right) \epsilon^{2}
\end{aligned}
$$

Since $c_{1}, c_{2}, c_{3}, d_{1}, d_{2}, d_{3}$ are positive constants, then $\dot{V}_{C 1}<0$ which means system (56) is stable. Proof of bounded output is given in [18].

Case 1.

Case 2: $x>0$ and $i_{1}=0$. In this case, the stability analysis can be treated in the same manner as in

\section{SIMULATION RESULTS}

The closed-loop system is numerically tested to verify the ability of the proposed control design. The AMB used in the numerical simulation has the following parameters:

\begin{tabular}{||c|c||}
\hline Parameters & Value \\
\hline \hline Nominal air-gap & $0.001 \mathrm{~m}$ \\
\hline Number of turns & 400 \\
\hline Coil resistance & $1 \Omega$ \\
\hline Cross-section area & $0.001 \mathrm{~m}^{2}$ \\
\hline Rotor mass & $2.6 \mathrm{Kg}$ \\
\hline Rotor initial position & $0.0004 \mathrm{Kg}$ \\
\hline Air-gap permeability & $1.256 .10^{-6}$ \\
\hline
\end{tabular}

Assume, initially the rotor is at a distance of $0.0004 \mathrm{~m}$ away from the $x_{0}$ ie. attached to coil 1 . The simulation is carried out in two scenarios. First, a conventional direct Lyapunov is applied and then Lyapunov function with appended term to limit system output response is embedded into the system.

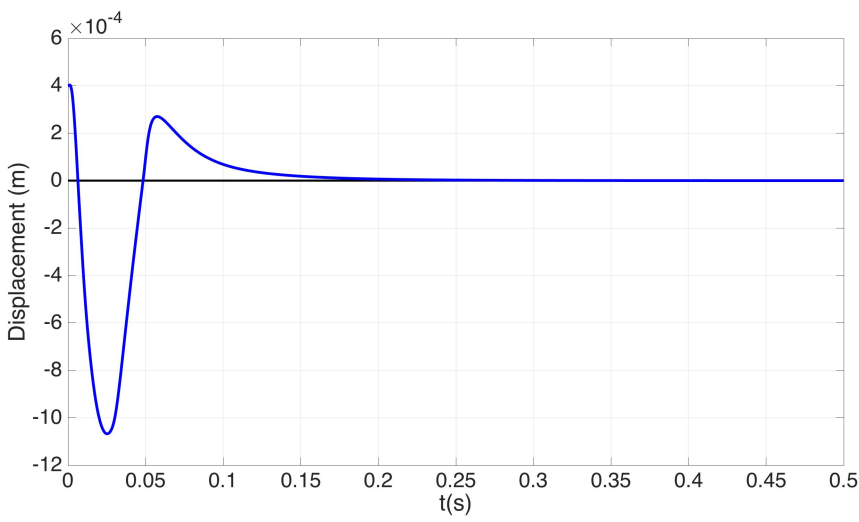

Figure 2. Displacement. 


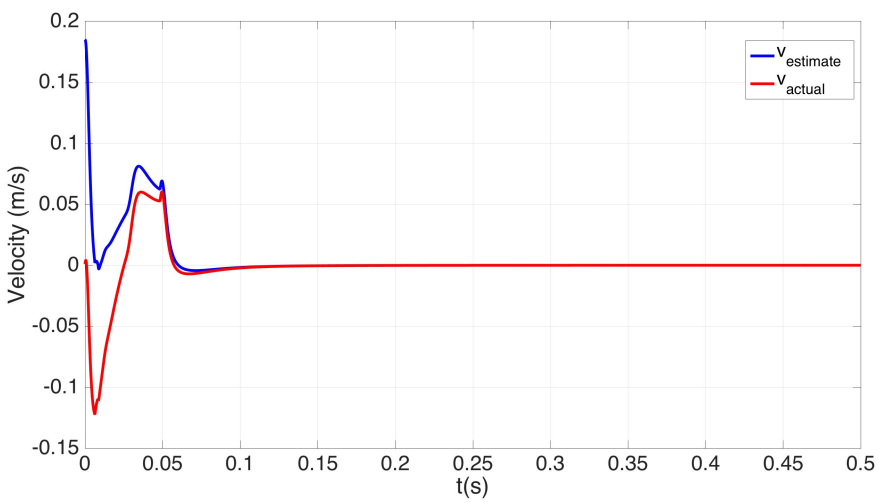

Figure 3. Actual and estimated velocity.

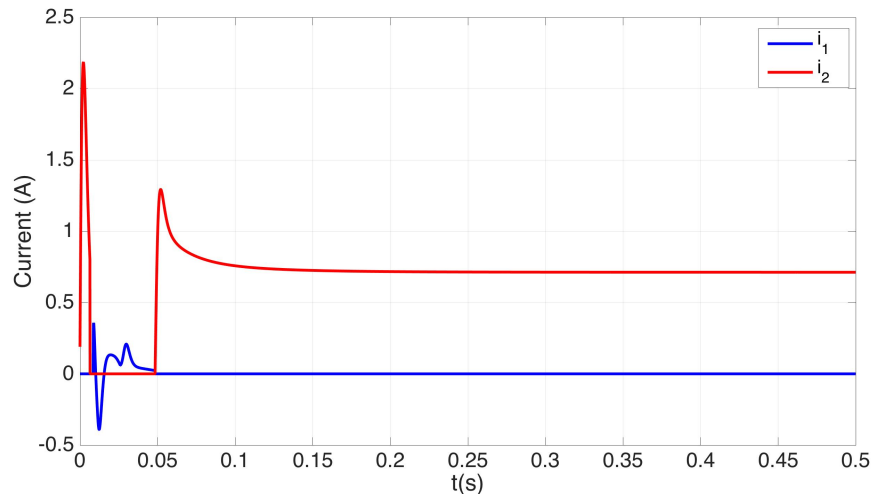

Figure 4. Current.

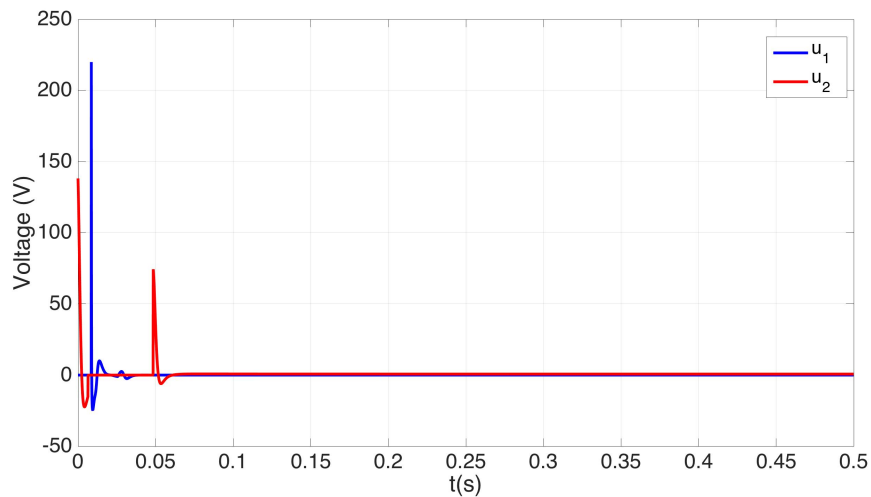

Figure 5. Control voltage.

It can be seen from the system responses that the rotor displacement has a maximum value of $0.001 \mathrm{~m}$. The phenomenon implies the rotor hits coil 2 during transient period, this is undesirable in practice. 


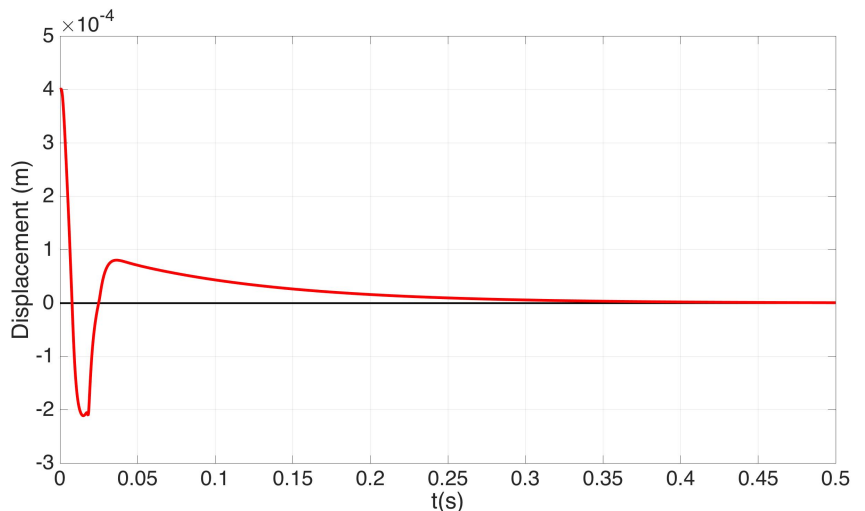

Figure 6. Displacement.

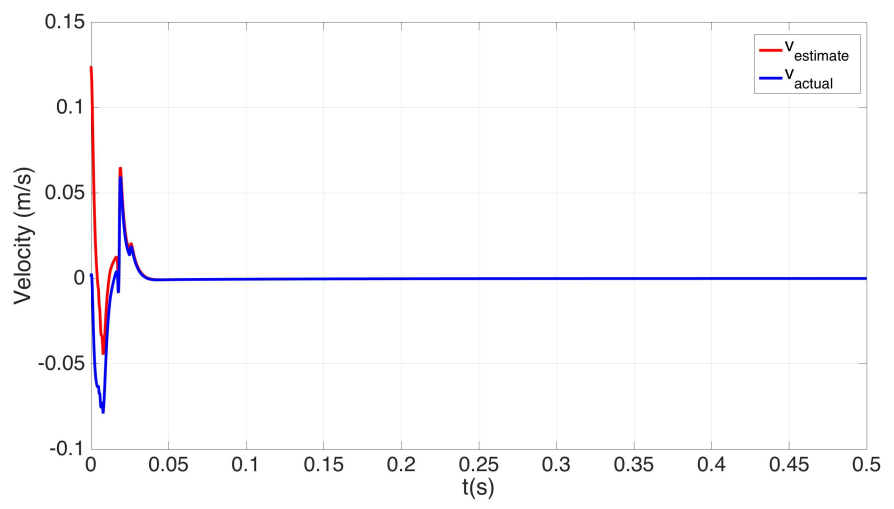

Figure 7. Actual and estimated velocity.

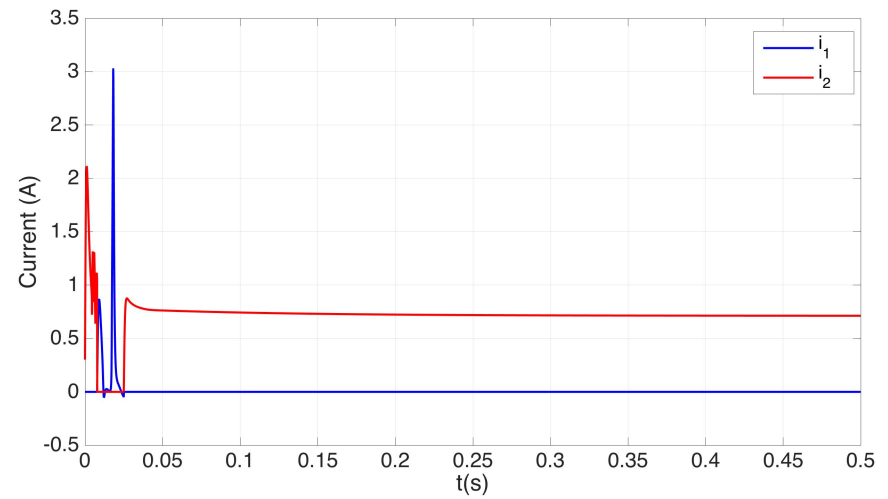

Figure 8. Current. 


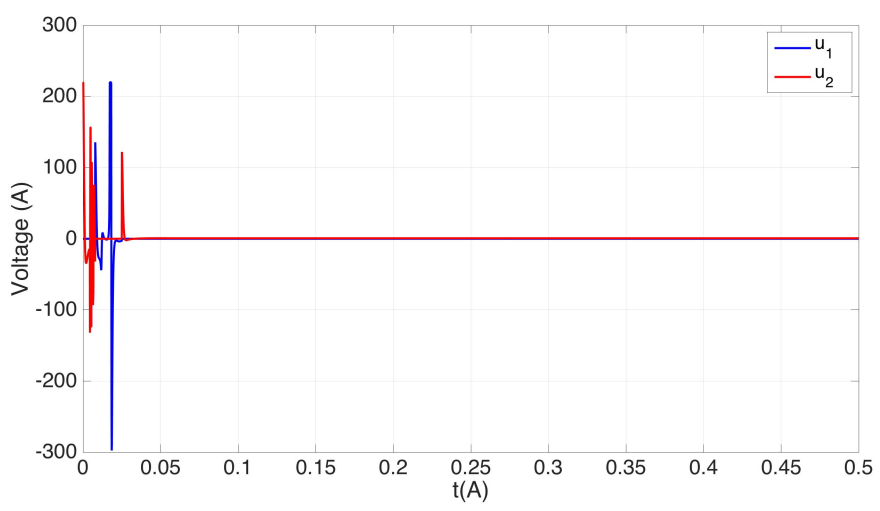

Figure 9. Control voltage.

In order to maintain the rotor position in safe range, the barrier Lyapunov candidate function is employed instead of the conventional one. System responses indicate better tracking performance. The rotor is kept in the predefined zone and possible mechanical contact with stator is avoided. In addition, the closed-loop system dynamics also prove the ability of the speed observer. It is noted that the control current and voltage are in a practical area.

\section{CONCLUSION}

This paper presents the positioning control design for an AMB system. To handle the inherent instability and nonlinearity of the AMB, back stepping control technique is employed. Besides, by introducing a barrier Lyapunov function in control design, the mechanical contact which may happen in transient response can be avoided. An nonlinear velocity is also used to reduce the cost of the system. The feasibility and effectiveness of the proposed control strategy are verified by simulations results. The simulations show good tracking performances of the system and all control inputs are in applicable values.

\section{REFERENCES}

[1] Hui. L, Yingjie. Y. (2012). "Bearing Fault Diagnosis Based on Laplace Wavelet Transform”, TELKOMNIKA, Vol.10, No.8, December 2012, pp. 21392150.

[2] Xinghui. Z, Jianshe. K, Jinsong. Z, 3, Hongzhi. T. (2014). "Bearing Run-To-Failure Data Simulation for Condition Based Maintenance", TELKOMNIKA, Vol.12, No.1, January 2014, pp. 514519.

[3] Normaisharah. M, Kasrul Abdul. K, Zulkiflie. Ibrahim, Tole. S, Siti A. A. T, Auzani. J. (2015). ”Bearingless Permanent Magnet Synchronous Motor using Independent Control", International Journal of Power Electronics and Drive System, Vol. 6, No. 2, June 2015, pp. 233241.

[4] Abulrub, A. (2006). "Modelling and control of contact in magnetic bearing/flexible rotor systems", Ph.D. thesis.

[5] Kang, C.S. (2014). "Control of an Active Magnetic Bearing- Rotor System", Ph.D. thesis.

[6] Aeschlimann, B. (2002). "Control Aspects of High Precision Active. Arbeit", 2489(2001).

[7] Farmakopoulos, M.G., Thanou, M.D., and Nikolakopou-los, P.G. (2013). ”A Control Model of Active Magnetic Bearings", In International Conference of Engineering Against Failure, June, 2628.

[8] Bronislaw, T., Dawid, W. and Jan, Z. (2011) "Modelling of Control System for an Active Magnetic Bearing", Proceedings of electrotechnical Institute, 252.

[9] Jastrzebski, R.P. and Pollanen, R. (2009). "Centralized optimal position control for active magnetic bearings: Comparison with decentralized control”, Electrical Engineering, 91(2), 101114. doi:10.1007/s00202-0090121-2.

[10] Kang, M.S., Lyou, J., and Lee, J.K. (2010). "Sliding mode control for an active magnetic bearing system subject to base motion", Mechatronics, 20(1), 171178. doi: 10.1016/j.mechatronics.2009.09.010.

[11] Tsai, N.C., Wu, B.Y., and Hsu, S.L. (2007). Stability constraints of active magnetic bearing control systems. International Journal of Control, 80(12), 18931902. doi:10.1080/00207170701447379.

[12] Noshadi, A., Shi, J., Lee, W.S., Shi, P., and Kalam, A. (2017). "Robust control of an active magnetic bearing system using $H_{\infty}$ and disturbance observer-based control", Journal of Vibration and Control, 23(11), 
18571870.

[13] Hsu, C.T. and Chen, S.L. (2002). "Exact linearization of a voltage-controlled 3-pole active magnetic bearing system", IEEE Transactions on Control Systems Technology, 10(4), 618625. doi:10.1109/TCST.2002.1014681.

[14] Lum, K.Y., Coppola, V.T., and Bernstein, D.S. (1996). "Adaptive autocentering control for an active magnetic bearing supporting a rotor with unknown mass imbalance", IEEE Transactions on Control Systems Technology, 4(5), 587597.

[15] Du, H., Zhang, N., Ji, J.C., and Gao, W. (2010). "Robust Fuzzy Control of an Active Magnetic Bearing Subject to Voltage Saturation", IEEE Transactions on Control Systems Technology, 18(1), 164169.

[16] Kim, H.Y. and Lee, C.W. (2006). "Design and control of active magnetic bearing system with Lorentz force- type axial actuator", Mechatronics, 16(1), 1320. doi: 10.1016/j.mechatronics.2005.09.005.

[17] Sivrioglu, S. (2007). Adaptive backstepping for switching control active magnetic bearing system with vibrating base. IET Control Theory Applications, 1(4), 1054 1059. doi:10.1049/iet-cta:20050473.

[18] Tee, K. P., et al (2009). Barrier Lyapunov Functions for control of output-constrained nonlinear systems. Automatica, 1(4), 1054 1059. doi:10.1016/j.automatica.2008.11.017.

\section{BIOGRAPHY OF AUTHORS}

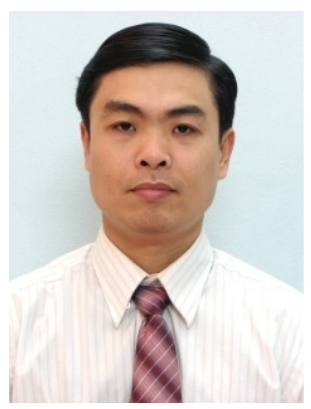

Danh Huy Nguyen received the Master degree in Control and Automation Engineering from Hanoi University of Science and Technology, Hanoi, Vietnam, 2000. He is current working toward a Ph.D at Department of Industrial Automation, School of Electrical Engineering, Hanoi University of Science and Technology. His research interests include motion control, magnetic bearing systems and applications.

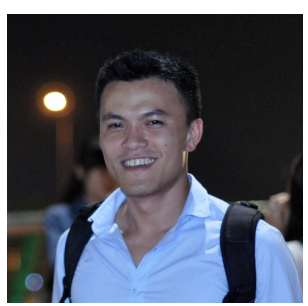

Tung Lam Nguyen received the B.S degree in Control and Automation Engineering from Hanoi University of Science and Technology, Hanoi, Vietnam, 2005, the M.S degree from Asian Institute of Technology, 2007, and the Ph.D from The University of Western Australia, 2014. He is current working as a lecturer at Department of Industrial Automation, School of Electrical Engineering, Hanoi University of Science and Technology. His research interests include motion control, control system and its applications.

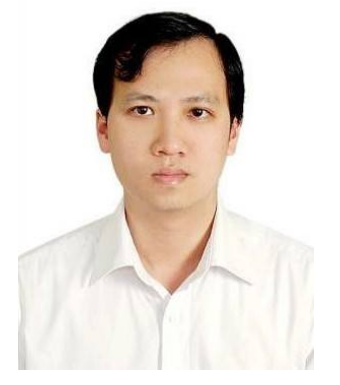

Manh Linh Nguyen received the B.S degree in Control and Automation Engineering from Hanoi University of Science and Technology, Hanoi, Vietnam, 2008 and the M.S degree from Graduate School of Engineering and Science in Shibaura Institute of Technology, Tokyo, Japan, 2015. From September 2008 to August 2014, he worked as an lecturer in the Department of Industrial Automation, School of Electrical Engineering, Hanoi University of Science and Technology, Hanoi, Vietnam. From September 2014 to August 2015, he was a M.S student and since September 2015 he has been a Ph.D student in Functional Control System Laboratory, Shibaura Institute of Technology, Tokyo, Japan. His research interests include model predictive control, sliding mode control, disturbance observer, advanced control theory and its applications to micro/nano positioning systems, digital control design for power electronic converters.

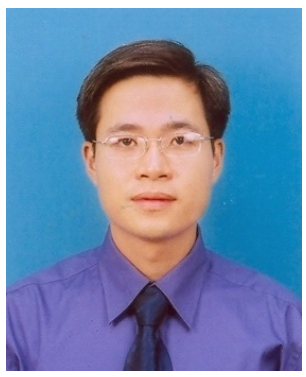

Huy Phuong Nguyen received the Ph.D degree in Control and Automation Engineering in 2000 $\mathrm{He}$ is the Head of School of Electrical Engineering, Hanoi University of Science and Technology. $\mathrm{He}$ interests in process control and systems theory. 